\title{
Algebraic convergence of function groups
}

\author{
Gero Kleineidam and Juan Souto
}

\begin{abstract}
We give a sufficient condition for a sequence of convex cocompact hyperbolic structures on a fixed compression body to have an algebraically convergent subsequence. This extends a result of Otal. Further if the manifold is a handlebody we show that certain laminations play a similar role in deformation space as binding curves in Teichmüller theory.
\end{abstract}

Mathematics Subject Classification (2000). 30F40, 20E08, 57M50.

Keywords. Hyperbolic manifolds, compression bodies, algebraic convergence, Masur domain, $\mathbb{R}$-trees.

\section{Introduction}

A compression body $N$ is a compact 3-manifold which is the connected sum along the boundary on a closed ball of solid tori and trivial interval bundles over closed surfaces of genus at least 2 . Throughout the paper, we only consider the case that the fundamental group $\pi_{1}(N)$ splits as a non-trivial free product. Equivalently, we rule out that $N$ is a trivial interval bundle over a closed surface or a solid torus. In particular, the boundary $\partial N$ has a unique compressible component which is called the exterior boundary $\partial_{e} N$. For more on the topology of compression bodies see Bonahon [Bon83, Appendix B].

Using Klein-combination one can construct a convex cocompact representation $\rho_{0}$ of $\pi_{1}(N)$ into $\mathrm{PSL}_{2} \mathbb{C}$ such that $\mathbb{H}^{3} / \rho_{0}\left(\pi_{1}(N)\right)$ is homeomorphic to the interior of the compression body $N$ [MT98]. Such a representation is said to uniformize $N$. The exterior boundary $\partial_{e} N$ is covered by a connected component of the discontinuity domain $\Omega_{\rho_{0}} \subset \hat{\mathbb{C}}$, which is invariant under $\rho_{0}\left(\pi_{1}(N)\right)$. Kleinian groups having an invariant component of the discontinuity domain are called function groups. The quotient of $\mathbb{H}^{3}$ by any convex cocompact function group is homeomorphic to the interior of a compression body. See Maskit [Mas88] for more on function groups.

Due to a theorem of Marden [And98, MT98], every quasi-conformal deformation of $\rho_{0}$ uniformizes $N$, too. By Ahlfors-Bers theory, $Q H\left(\rho_{0}\right)$, the space of

The authors were partially supported by the Sonderforschungsbereich 256 . 
quasi-conformal deformations of $\rho_{0}$ up to conjugation by an element of $\mathrm{PSL}_{2} \mathbb{C}$, is parameterized by the Teichmüller space $\mathcal{T}(\partial N)$. More precisely, there is a normal covering, called the Ahlfors-Bers map

$$
\mathcal{T}(\partial N) \rightarrow Q H\left(\rho_{0}\right)
$$

whose deck transformation group is the group of isotopy classes of diffeomorphisms of $N$ which are homotopic to the identity (see [MT98]). Note that the Teichmüller space $\mathcal{T}(\partial N)$ can be identified with $\mathcal{T}\left(\partial_{e} N\right) \times \mathcal{T}\left(\partial_{1} N\right) \times \cdots \times \mathcal{T}\left(\partial_{k} N\right)$ where $\partial_{e} N, \partial_{1} N, \ldots, \partial_{k} N$ are the boundary components of $N$. As homeomorphisms of $N$ preserve $\partial_{e} N$, the deck transformation group of the Ahlfors-Bers map acts on $\mathcal{T}\left(\partial_{e} N\right)$.

The space $Q H\left(\rho_{0}\right)$ is contained in the deformation space of $\pi_{1}(N)$, the space of $\mathrm{PSL}_{2} \mathbb{C}$-conjugacy classes of discrete and faithful representations of $\pi_{1}(N)$ into $\mathrm{PSL}_{2} \mathbb{C}$. The compact-open topology on it is the so-called algebraic topology. With respect to this topology, $Q H\left(\rho_{0}\right)$ is open in deformation space. For more on the deformation theory of Kleinian groups, see [And98, MT98].

Since deformation space is not compact, it is an interesting question to determine when a divergent sequence in $Q H\left(\rho_{0}\right)$ converges in deformation space.

Canary [Can91] showed that for all compact subsets $K \subset \mathcal{T}\left(\partial_{e} N\right)$ the image under the Ahlfors-Bers map of $K \times \mathcal{T}\left(\partial_{1} N\right) \times \cdots \times \mathcal{T}\left(\partial_{k} N\right)$ has compact closure in deformation space. The goal of this paper is to study sequences of quasi-conformal deformations of $\rho_{0}$ such that the corresponding sequences in $\mathcal{T}\left(\partial_{e} N\right)$ diverge.

Thurston [FLP79] compactified Teichmüller space via $\mathcal{P} \mathcal{M L}$, the space of projective classes of measured laminations. Masur [Mas86] and Otal [Ota88] studied the dynamics of the mapping class group of the compression body $N$ on the space of projective classes of measured laminations on the exterior boundary $\partial_{e} N$ and described an open set $\mathcal{O} \subset \mathcal{P} \mathcal{M L}$ on which the action is properly discontinuous. The set $\mathcal{O}$ is called the Masur domain. Otal [Ota88] analyzed further geometric properties of laminations in $\mathcal{O}$. In particular, he proved that given a convex cocompact representation uniformizing $N$, every lamination $\lambda$ in the Masur domain is realized by a pleated surface.

We will say that a sequence $\left(\rho_{i}\right)$ in $Q H\left(\rho_{0}\right)$ converges into the Masur domain if it is parameterized under the Ahlfors-Bers map by a sequence $\left(S_{i}^{e}, S_{i}^{1}, \ldots, S_{i}^{k}\right)_{i} \subset$ $\mathcal{T}(\partial N)$ such that $\left(S_{i}^{e}\right)_{i}$ converges to a measured lamination $\lambda \in \mathcal{O}$. We impose no restrictions on the conformal structures on the incompressible boundary components. By abuse, we will say that the sequence $\left(\rho_{i}\right) \subset Q H\left(\rho_{0}\right)$ converges to $\lambda \in \mathcal{O}$.

Conjecture (Thurston). Let $\rho_{0}$ be a convex cocompact representation uniformizing a compression body $N$. If $\left(\rho_{i}\right)_{i}$ is a sequence in $Q H\left(\rho_{0}\right)$ converging to a lamination in the Masur domain, then it has a convergent subsequence in deformation space.

Canary [Can93] proved the conjecture under the extra assumption that $N$ is a handlebody and that there is an identification of $N$ with the trivial interval bundle 
over a surface $\Sigma$ with boundary $\partial \Sigma$ such that the lengths of the geodesics in the free homotopy classes of $\partial \Sigma$ remain bounded with respect to the representations $\rho_{i}$.

In case $N$ is the connected sum along the boundary of two trivial bundles over closed surfaces, Ohshika [Ohs97] gave a partial answer to the above conjecture.

Otal [Ota94] proved that the conjecture holds for handlebodies of genus two and laminations with simply connected complementary regions. Such laminations are also called minimal arational. We follow the strategy of Otal's proof and show for general compression bodies.

Theorem 1. Let $\rho_{0}$ be a convex cocompact representation uniformizing a compression body $N$. If $\left(\rho_{i}\right)_{i}$ is a sequence in $Q H\left(\rho_{0}\right)$ converging to a minimal arational lamination in the Masur domain, then it has a convergent subsequence in deformation space.

Further, we prove that laminations in the Masur domain play a similar role for handlebodies as binding curves do in Teichmüller theory. This generalizes Theorem 6.1 in Canary [Can93].

Theorem 2. Let $N$ be a handlebody and $\lambda$ a measured lamination in the Masur domain. The set of convex cocompact representations $\rho$ uniformizing $N$ such that $l_{\rho}(\lambda)$, the length of $\lambda$ with respect to $\rho$, is less than a constant $C>0$ is precompact in deformation space.

The proofs of Theorem 1 and Theorem 2 follow the same lines. We restrict ourselves to a brief outline of the proof of Theorem 1 .

Seeking for a contradiction, let $\left(\rho_{i}\right)$ be a sequence in $Q H\left(\rho_{0}\right)$ which converges to a minimal arational lamination $\lambda \in \mathcal{O}$ but does not contain any convergent subsequence in deformation space.

By Theorems of Thurston [FLP79] and Canary [Can91], convergence to $\lambda$ implies that there is a sequence of curves $\left(\gamma_{i}\right)$ on $\partial_{e} N$ converging to $\lambda$ in $\mathcal{P} \mathcal{M L}$ such that the ratios of the translation lengths in $\mathbb{H}^{3}$ of $\rho_{i}\left(\gamma_{i}\right)$ and $\rho_{0}\left(\gamma_{i}\right)$ tend to 0.

By a Theorem of Morgan and Shalen [MS84], divergence in deformation space implies that a subsequence of $\left(\rho_{i}\right)$, say the whole sequence, converges in an appropriate sense to a minimal and small action of $\pi_{1}(N)$ on an $\mathbb{R}$-tree $T$. The lamination $\lambda$ is said to be realized in $T$ if there is a continuous equivariant map from $\tilde{\lambda}$, the lift of $\lambda$ to $\mathbb{H}^{2}$, to $T$ which is injective on each leaf of $\tilde{\lambda}$. Otal [Ota94] proved that if $\lambda$ is realized in $T$, then for every sequence of curves $\left(\gamma_{i}\right)$ converging to $\lambda$ in $\mathcal{P} \mathcal{M L}$, the ratios of the translation lengths in $\mathbb{H}^{3}$ of $\rho_{i}\left(\gamma_{i}\right)$ and $\rho_{0}\left(\gamma_{i}\right)$ tend to $\infty$.

Hence, we obtain the desired contradiction by proving that every minimal arational lamination in the Masur domain is realized in every $\mathbb{R}$-tree which admits a minimal small action of $\pi_{1}(N)$. This was previously established by Otal [Ota94] in the case of the handlebody of genus 2. He made use of a Theorem of Culler and Vogtmann [CV91] which gives a geometric description of all small actions on $\mathbb{R}$-trees of the free group of rank 2 . It is known that such a characterization is not 
possible in general, not even for free groups of higher rank [GL95].

Our approach is different. Suppose that a minimal arational lamination $\lambda \in \mathcal{O}$ and an $\mathbb{R}$-tree $T$ that admits a small action of $\pi_{1}(N)$ are given. We show that there is a measured lamination $\mu$ on the exterior boundary $\partial_{e} N$ and a morphism from the dual tree $T_{\mu}$ to $T$ such that the composition of the projection $\mathbb{H}^{2} \rightarrow T_{\mu}$ with the morphism $T_{\mu} \rightarrow T$ is monotone and non-constant on every leaf of $\tilde{\lambda}$. Due to a result of Otal [Ota94], such a map can be homotoped to a realization of $\lambda$ in $T$.

The existence of $\mu$ ensues from a detailed study of morphisms from dual trees to $T$. This part is motivated by ideas of Skora [Sko96].

The paper is structured as follows: In section 2, we review some facts about trees, laminations, and divergence of representations. In Section 3, we reduce Theorem 1 and Theorem 2 to statements on realizations of laminations in $\mathbb{R}$-trees. Section 4 is devoted to the analysis of laminations on the exterior boundary of $N$. The techniques are motivated by earlier work of Otal [Ota88]. In section 5 , we study morphisms from dual trees to $\mathbb{R}$-trees which admit a minimal small action of $\pi_{1}(N)$. In section 6 , we construct the desired realizations using the results of section 4 and section 5 .

The authors would like to express their special gratitude to Professor Ursula Hamenstädt and Professor Jean-Pierre Otal for their patience, their encouragement and the fruitful discussions with them. There is no doubt that the present paper would not have been possible without Otal's fundamental work on this topic. The first author wants to thank Professor Frédéric Paulin for his invitation to a two-month-stay in Orsay.

We thank the referee for a careful reading and useful suggestions.

\section{Preliminaries}

In this section, we review some facts about trees, laminations and divergence of representations.

Let $N$ be a compression body. After the choice of a basepoint $\star$ on the exterior boundary $\partial_{e} N$, we have a surjective homomorphism

$$
\varphi: \pi_{1}\left(\partial_{e} N, \star\right) \rightarrow \pi_{1}(N, \star)
$$

which we call compression homomorphism. If there is no risk of confusion, we will use the symbol $\gamma$ for elements in $\pi_{1}\left(\partial_{e} N, \star\right)$ as well as for their images under $\varphi$. Further for simplicity, we often write $\pi_{1}\left(\partial_{e} N\right)$ and $\pi_{1}(N)$.

Later it will be useful to view every action $\pi_{1}(N) \curvearrowright X$ of $\pi_{1}(N)$ on a space $X$ as a $\pi_{1}\left(\partial_{e} N\right)$-action, too:

$$
\pi_{1}\left(\partial_{e} N\right) \times X \rightarrow X, \quad(g, x) \mapsto \varphi(g) x
$$

All actions on metric spaces $\left(X, d_{X}\right)$ will be isometric. The translation length of an isometry $g$ of a metric space $X$ is defined to be $\inf \left\{d_{X}(x, g x) \mid x \in X\right\}$. The metric spaces we are going to work with are $\mathbb{H}^{2}, \mathbb{H}^{3}$ and $\mathbb{R}$-trees. 


\section{1. $\mathbb{R}$-trees}

An $\mathbb{R}$-tree $T$ is a path metric space such that any two points $p, q$ can be joined by a unique arc.

There is a classification of the isometries of an $\mathbb{R}$-tree $T$. An isometry $g: T \rightarrow T$ has either translation length $l_{T}(g)=0$ and has a fixed point or $l_{T}(g)>0$ and there is a unique invariant geodesic line in $T$, the axis of $g$.

An action $G \curvearrowright T$ is called minimal if there is no proper invariant subtree. An action $G \curvearrowright T$ is called small if the stabilizer of every non-degenerate arc is virtually abelian. More about $\mathbb{R}$-trees can be found in Kapovich [Kap00].

Morgan and Shalen [MS84] used $\mathbb{R}$-trees to compactify the deformation space. They use algebraic methods, for a more geometric approach see Bestvina [Bes88] and Paulin [Pau88].

Compactness Theorem (Morgan-Shalen). Let $G$ be a finitely generated group containing a free group of rank 2 and let $\rho_{i}: G \rightarrow \mathrm{PSL}_{2} \mathbb{C}$ be a sequence of discrete and faithful representations. Then after passing to a subsequence either

(1) $\left(\rho_{i}\right)$ converges in the deformation space of $G$, or

(2) there is a minimal small action $G \curvearrowright T$ on an $\mathbb{R}$-tree and a sequence of real numbers $\epsilon_{i} \rightarrow 0$ with

$$
\lim _{i} \epsilon_{i} l_{\rho_{i}}(\gamma)=l_{T}(\gamma)
$$

for all $\gamma \in G$, where $l_{\rho_{i}}(\gamma)$ is the translation length of $\rho_{i}(\gamma)$ in $\mathbb{H}^{3}$.

We remark that a minimal small action of such a group $G$ on a tree is characterized by the translation lengths of the elements of $G$ [Kap00]. This allows us to say that the sequence $\left(\rho_{i}\right)_{i}$ converges to the action $G \curvearrowright T$.

We will apply the Compactness Theorem to the case that $G$ is the fundamental group of a compression body.

\subsection{Laminations}

A lamination on a closed hyperbolic surface $S$ is a compact subset of $S$ which can be decomposed as a disjoint union of simple geodesics, called leaves. The sets of laminations with respect to two hyperbolic structures on the same surface can be naturally identified (see [CB88]); so a lamination can be considered as a topological object. A lamination is called minimal if every half-leaf is dense. Each lamination can be decomposed as a union of finitely many connected minimal laminations, called minimal components, and finitely many non-compact isolated leaves. The set of laminations is compact with respect to the topology induced by the Hausdorff distance. We will refer to this topology as the Hausdorff topology. 
A train-track $\tau$ in $S$ is a finite union of rectangles with "long" horizontal sides which are foliated by "short" vertical geodesic segments and which meet only at non-degenerate segments contained in the vertical sides. The horizontal sides of the rectangles are called rails, the vertical segments ties and the intersections of two rectangles switches. A lamination is carried by $\tau$ if it is contained in $\tau$ and transverse to the ties. If $\lambda$ is carried by $\tau$, then the set of laminations carried by $\tau$ forms a neighbourhood of $\lambda$ with respect to the Hausdorff topology [Ota96].

A measured lamination is a lamination with a transverse measure of full support. The support of a measured lamination is a finite union of minimal components, in particular it does not contain any isolated non-compact leaf. A minimal lamination is called minimal arational if its complementary regions are simplyconnected.

There is a topology on the set $\mathcal{M L}$ of measured laminations which is induced by the intersection form $i: \mathcal{M L} \times \mathcal{M L} \rightarrow \mathbb{R}_{+}$[Ota96, FLP79]. Rescaling the measure provides an action of $\mathbb{R}_{+}$on $\mathcal{M L}$. The quotient with the quotient topology is the space of projective measured laminations and is denoted $\mathcal{P} \mathcal{M L}$. It is compact. If a sequence of projective measured laminations converges to a projective measured lamination $\lambda$ in $\mathcal{P} \mathcal{M L}$ and to a lamination $\lambda_{\mathcal{H}}$ in the Hausdorff topology, then $\lambda$ - or more precisely the support of $\lambda$ - is contained in $\lambda_{\mathcal{H}}$.

The Teichmüller space of a closed surface $S$ is denoted by $\mathcal{T}(S)$. Thurston [Thu86] studied the length function on the space of measured laminations on $S$. It is the unique continuous function

$$
\mathcal{T}(S) \times \mathcal{M L} \longrightarrow \mathbb{R}_{+}, \quad(\sigma, \lambda) \mapsto l_{\sigma}(\lambda)
$$

which extends the function that associates to a point $\sigma \in \mathcal{T}(S)$ and to a weighted simple closed geodesic $a \cdot \gamma, a>0$, the length of $\gamma$ in $\sigma$ multiplied by $a$.

The Teichmüller space $\mathcal{T}(S)$ can be compactified by the space $\mathcal{P} \mathcal{M L}$ of projective measured laminations on $S$ [FLP79]. This compactification reflects the geometric behaviour of divergent sequences in $\mathcal{T}(S)$. In particular, if a sequence $\left(S_{i}\right) \subset \mathcal{T}(S)$ converges to $\lambda \in \mathcal{P} \mathcal{M L}$, then there is a sequence $\left(\gamma_{i}\right)$ of simple closed curves converging to $\lambda$ in $\mathcal{P} \mathcal{M L}$ and such that

$$
l_{S_{i}}\left(\gamma_{i}\right) / l_{S_{0}}\left(\gamma_{i}\right) \longrightarrow 0 \text { for } i \longrightarrow \infty \text {. }
$$

However this does not imply that the lengths $l_{S_{i}}(\lambda)$ tend to 0 . Indeed, take an element of $\mathcal{T}(S)$ and iterate a Dehn twist about a fixed curve $\gamma$ on it. The resulting sequence tends to $\gamma$, seen as an element of $\mathcal{P} \mathcal{M L}$, but the length of $\gamma$ is constant during the sequence.

Given a hyperbolic structure on the compression body $N$, a pleated surface is a length preserving map $f: S \rightarrow N$ from a hyperbolic surface $S \in \mathcal{T}\left(\partial_{e} N\right)$ to $N$ homotopic to the inclusion $\partial_{e} N \hookrightarrow N$ and such that each point $p \in S$ is contained in a geodesic segment which is mapped isometrically. A lamination $\lambda$ on the exterior boundary $\partial_{e} N$ is realized by a pleated surface if there is a pleated surface that maps each leaf of $\lambda$ to a geodesic in $N$. Notice that a realization of a lamination $\lambda$ by a pleated surface induces a map from $\lambda$ to the projectivized 
tangent bundle of $N$.

If $\rho$ is a representation of $\pi_{1}(N)$ into $\mathrm{PSL}_{2} \mathbb{C}$ uniformizing $N$ and $\lambda$ is a measured lamination on the exterior boundary that is realized by a pleated surface, we can define $l_{\rho}(\lambda)$, the length of $\lambda$ with respect to $\rho$, to be its length with respect to the hyperbolic structure of the pleated surface realizing $\lambda$.

See Otal [Ota96, Appendix] for more about laminations and measured laminations and Fathi-Laudenbach-Poénaru [FLP79] for a detailed exposition of Thurston's compactification of Teichmüller space. Pleated surfaces are discussed in [Thu86, Ota88, Kap00].

\subsection{Dual trees}

For a measured lamination $\mu$ on a surface $S$, we denote by $\tilde{\mu}$ its lift to $\mathbb{H}^{2}$. If $\mu$ does not have atoms, the semidistance on $\mathbb{H}^{2}$ induced by integrating the transverse measure $\tilde{\mu}$ along paths is continuous with respect to the usual topology of $\mathbb{H}^{2}$. The support of an atom is a closed geodesic, therefore it is possible to avoid atoms by replacing closed leaves in $\mu$ by annuli foliated by parallel closed curves. Denote by $\mathcal{F}_{\mu}$ the measured partial foliation that we obtain by this process and by $\widetilde{\mathcal{F}}_{\mu}$ the lift of $\mathcal{F}_{\mu}$ to $\mathbb{H}^{2}$. The quotient of $\mathbb{H}^{2}$ under the semi-distance induced by $\mathcal{F}_{\mu}$ depends only on $\mu$, it is denoted $T_{\mu}$, and the projection $\pi_{\mathcal{F}_{\mu}}: \mathbb{H}^{2} \rightarrow T_{\mu}$ is continuous. $T_{\mu}$ is an $\mathbb{R}$-tree, called the dual tree of $\mu$. The fundamental group of the surface $S$ acts on $T_{\mu}$ and the action is small and minimal. Dual trees are discussed in detail by Otal [Ota96] and Kapovich [Kap00].

Using dual trees, Skora [Sko96] established a 1-1-correspondence between minimal small actions of the fundamental group of a closed surface $S$ and measured laminations on $S$.

Theorem (Skora). Let $\pi_{1}(S) \curvearrowright T$ be a minimal and small action of $\pi_{1}(S)$ on an $\mathbb{R}$-tree $T$, then there is a unique $\mu \in \mathcal{M L}$ and an equivariant isometry $T_{\mu} \rightarrow T$.

Skora's ideas will be used in section 5 where we study certain maps from trees dual to laminations on the exterior boundary of a compression body $N$ to a given $\mathbb{R}$-tree with a minimal small action of $\pi_{1}(N)$.

\section{Main Theorems}

The goal of this section is to reduce Theorem 1 and Theorem 2 to a property of minimal small actions of the fundamental group of a compression body $N$ on $\mathbb{R}$-trees.

A simple closed curve $m$ on the exterior boundary $\partial_{e} N$ which is homotopically trivial in $N$ but not in $\partial_{e} N$ is called a meridian. Note that by Dehn's Lemma 
[Jac80] every meridian bounds an embedded disk in $N$. Recall that we use the symbol $\gamma$ for elements in $\pi_{1}\left(\partial_{e} N\right)$ as well as for their images under the compression homomorphism $\varphi: \pi_{1}\left(\partial_{e} N\right) \rightarrow \pi_{1}(N)$.

A meridian may be seen as an element of $\mathcal{P} \mathcal{M L}$, too. The set of projective classes of weighted multicurves of meridians in $\mathcal{P} \mathcal{M L}$ will be denoted by $M$ and its closure in $\mathcal{P} \mathcal{M L}$ by $M^{\prime}$ (see Otal [Ota88]).

$N$ is a small compression body if it is the connected sum along the boundary of either two trivial interval bundles over closed surfaces or an interval bundle over a closed surface and a solid torus. For a small compression body, set

$$
\begin{gathered}
\mathcal{O}:=\{\lambda \in \mathcal{P} \mathcal{M L} \mid i(\lambda, \mu)>0 \text { for all } \mu \in \mathcal{P} \mathcal{M L} \text { such that } \\
\text { there is } \left.\nu \in M^{\prime} \text { with } i(\mu, \nu)=0\right\}
\end{gathered}
$$

If $N$ is not a small compression body, set

$$
\mathcal{O}:=\left\{\lambda \in \mathcal{P} \mathcal{M L} \mid i(\lambda, \mu)>0 \text { for all } \mu \in M^{\prime}\right\}
$$

The set $\mathcal{O}$ is called the Masur domain and is open by continuity of the intersection form and compactness of $\mathcal{P} \mathcal{M L}$. We will say that $\lambda \in \mathcal{M L}$ is in $\mathcal{O}$ (resp. $M^{\prime}$ ) if its projective class is in $\mathcal{O}$ (resp. $M^{\prime}$ ).

Otal [Ota88] proved (see also Ohshika [Ohs])

Theorem on pleated surfaces (Otal). Let $N$ be a compression body with a convex cocompact hyperbolic structure. Every lamination $\lambda \subset \partial_{e} N$ containing the support of a measured lamination in the Masur domain is realized by a pleated surface in $N$.

Moreover, the induced map from $\lambda$ to the projectivized tangent bundle of $N$ is a homeomorphism onto its image $\mathcal{P}_{\lambda}$.

The image in $\mathcal{P}_{\lambda}$ of a leaf of $\lambda$ is the trace of a geodesic and is equally called a leaf. Denote by $\widetilde{\mathcal{P}}_{\lambda}$ the preimage of $\mathcal{P}_{\lambda}$ in the projectivized tangent bundle of $\mathbb{H}^{3}$. The following definition is due to Otal [Ota94]:

Definition. Let $N$ be a compression body with a convex cocompact hyperbolic structure and $\pi_{1}(N) \curvearrowright T$ an action on an $\mathbb{R}$-tree $T$. A lamination $\lambda \subset \partial_{e} N$ is realized in $T$ if there is a continuous $\pi_{1}(N)$-equivariant map

$$
\Phi: \widetilde{\mathcal{P}}_{\lambda} \rightarrow T
$$

which is injective when restricted to any leaf.

As $\lambda$ is mapped homeomorphically onto $\widetilde{\mathcal{P}}_{\lambda}$, this definition is equivalent to the definition of realization given in the introduction.

The following Theorem was proved by Otal [Ota94] in the case that $N$ is a handlebody, but a careful checking of the proof shows that it holds for compression bodies as well. 
Continuity Theorem (Otal). Let $\left(\rho_{i}\right)$ be a sequence in the deformation space of $\pi_{1}(N)$ converging to a non-trivial minimal small action $\pi_{1}(N) \curvearrowright T$ and $\left(\gamma_{i}\right)$ a sequence of simple closed curves on $\partial_{e} N$ converging in $\mathcal{P} \mathcal{M L}$ to a minimal arational measured lamination $\lambda$ in $\mathcal{O}$ which is realized in $T$. Then for all $N>0$ there is $i_{N}>0$ such that

$$
l_{\rho_{i}}\left(\gamma_{i}\right) \geq N l_{\rho_{0}}\left(\gamma_{i}\right) \text { for all } i \geq i_{N}
$$

where $l_{\rho}(\gamma)$ denotes the translation length of $\rho(\varphi(\gamma))$ in $\mathbb{H}^{3}$.

Remark. It seems to be a delicate issue to extend the theorem to all sequences of curves $\left(\gamma_{i}\right)$ converging in $\mathcal{P} \mathcal{M L}$ to an arbitrary lamination $\lambda$ in $\mathcal{O}$ which is realized in the tree $T$.

On the other hand, the conclusion of the theorem is valid for an arbitrary sequence of curves $\left(\gamma_{i}\right)$ provided every Hausdorff limit of $\left(\gamma_{i}\right)$ is realized in T. Indeed, the latter condition is the only one used in the proof of the theorem, and it is weaker than the one stated in the theorem (see [Ota94]).

In the last section we will prove

Theorem 3. Let $\pi_{1}(N) \curvearrowright T$ be a non-trivial minimal small action on an $\mathbb{R}$-tree $T$ and $\lambda$ a minimal arational measured lamination in the Masur domain, then $\lambda$ is realized in $T$.

Next we reduce Theorem 1 to Theorem 3 (see Otal [Ota94]).

Theorem 1. Let $\rho_{0}$ be a convex cocompact representation uniformizing a compression body $N$. If $\left(\rho_{i}\right)_{i}$ is a sequence in $Q H\left(\rho_{0}\right)$ converging to a minimal arational lamination in the Masur domain, then it has a convergent subsequence in deformation space.

Proof. Seeking a contradiction, suppose that $\left(\rho_{i}\right)_{i}$ converges to a minimal and small action $\pi_{1}(N) \curvearrowright T$ on an $\mathbb{R}$-tree. By definition, there is a sequence $\left(S_{i}^{e}, S_{i}^{1}, \ldots, S_{i}^{k}\right) \subset$ $\mathcal{T}(\partial N)$ which is mapped to $\left(\rho_{i}\right)$ under the Ahlfors-Bers map and such that the sequence $\left(S_{i}^{e}\right) \subset \mathcal{T}\left(\partial_{e} N\right)$ converges to $\lambda$. Then there is a sequence $\left(\gamma_{i}\right)$ of simple closed curves on the exterior boundary $\partial_{e} N$ converging to $\lambda$ in $\mathcal{P M L}$ with

$$
l_{S_{i}^{e}}\left(\gamma_{i}\right) / l_{S_{0}^{e}}\left(\gamma_{i}\right) \longrightarrow 0 \text { for } i \longrightarrow \infty .
$$

On the other hand, for all $A>0$ there is some $i_{A}$ such that $l_{S_{i}^{e}}(m)>A$ for all $i \geq i_{A}$ and all meridians $m$. Then by a Theorem due to Canary [Can91] there is $K>0$ such that for all $i \geq i_{A}$

$$
l_{\rho_{i}}\left(\gamma_{i}\right) \leq K l_{S_{i}^{e}}\left(\gamma_{i}\right)
$$

where $l_{\rho_{i}}\left(\gamma_{i}\right)$ is the translation length of $\rho_{i}\left(\varphi\left(\gamma_{i}\right)\right)$ in $\mathbb{H}^{3}$. Combining equation (1) and equation (2) we deduce with the same arguments as Canary [Can93] in the 
handlebody case that

$$
l_{\rho_{i}}\left(\gamma_{i}\right) / l_{\rho_{0}}\left(\gamma_{i}\right) \longrightarrow 0 \text { for } i \longrightarrow \infty
$$

By the Continuity Theorem, $\lambda$ is not realized in $T$, contradicting Theorem 3.

In the case that $N$ is a handlebody we will show in section 6

Corollary 3. Let $N$ be a handlebody and $\pi_{1}(N) \curvearrowright T$ be a non-trivial minimal small action on an $\mathbb{R}$-tree $T$. At least one minimal component of every measured lamination in the Masur domain is realized in $T$.

We reduce Theorem 2 to Corollary 3 .

Theorem 2. Let $N$ be a handlebody and $\lambda$ a measured lamination in the Masur domain. The set of convex cocompact representations $\rho$ uniformizing $N$ such that $l_{\rho}(\lambda)$, the length of $\lambda$ with respect to $\rho$, is less than a constant $C>0$ is precompact in deformation space.

Proof. Suppose again that there is a sequence $\left(\rho_{i}\right)$ of convex cocompact representations uniformizing $N$ with $l_{\rho_{i}}(\lambda)<C$ that converges to some non-trivial minimal small action $\pi_{1}(N) \curvearrowright T$ on an $\mathbb{R}$-tree. The length of any minimal component $\lambda_{0}$ of $\lambda$ is also bounded by $C$ for all $i$.

By Otal's Theorem on pleated surfaces, the lamination $\lambda$ is realized by a pleated surface in $\mathbb{H}^{3} / \rho_{i}\left(\pi_{1}(N)\right)$ for all $i$. Hence in each neighbourhood of $\lambda_{0}$ with respect to the Hausdorff topology we find a simple closed curve $\gamma_{i}$ with $l_{\rho_{i}}\left(\gamma_{i}\right)<C l_{\rho_{0}}\left(\gamma_{i}\right)$.

By a diagonal argument, we can assume that the sequence $\left(\gamma_{i}\right)$ converges to $\lambda_{0}$ in the Hausdorff topology.

The Continuity Theorem applies to this sequence by the remark after it; thus, the lamination $\lambda_{0}$ cannot be realized in $T$. As $\lambda_{0}$ was arbitrary this contradicts Corollary 3.

\section{Laminations on the exterior boundary}

Let $\rho_{0}: \pi_{1}(N) \rightarrow \mathrm{PSL}_{2} \mathbb{C}$ be a convex cocompact representation uniformizing the compression body $N$. The image of $\rho_{0}$ is a function group. Following Otal's [Ota88] notation, $S^{\prime} \subset \hat{\mathbb{C}}$ denotes the invariant component of the discontinuity domain of the action of $\rho_{0}\left(\pi_{1}(N)\right)$. It is a normal planar covering of the exterior boundary $\partial_{e} N$ with deck transformation group $\rho_{0}\left(\pi_{1}(N)\right)$. Since $\rho_{0}\left(\pi_{1}(N)\right)$ is a function group, the limit set $\Lambda_{\rho_{0}}$ of the action of $\rho_{0}\left(\pi_{1}(N)\right)$ on $\hat{\mathbb{C}}$ coincides with the boundary of $S^{\prime}$ in $\hat{\mathbb{C}}[\operatorname{Mas} 88]$. 


\subsection{Treelike structure of $S^{\prime}$}

We recall that a meridian is a simple closed curve which is nullhomotopic in $N$ but not in $\partial_{e} N$. By Dehn's Lemma, a meridian bounds an embedded disk in $N$. So, every maximal disjoint union of non-parallel meridians cuts $N$ into smaller pieces. On the level of groups, this means that $\pi_{1}(N)$ is a graph of groups whose edge groups are trivial and correspond to the meridians. The universal cover of the graph is a tree and there is a $\pi_{1}(N)$-equivariant map from $S^{\prime}$ to the tree. Such a map maps geodesics in $S^{\prime}$ to paths in the tree. In general, the paths can be fairly arbitrary, in particular not monotone.

Definition. Let $m$ be a meridian. An $m$-wave is an $\operatorname{arc}$ on $\partial_{e} N$ with endpoints on $m$ which is homotopic in $N$ relative endpoints, but not in $\partial_{e} N$ to a subarc of $m$.

For example, if two meridians intersect, then each of them contains a wave with respect to the other.

Definition. A curve $\gamma: \mathbb{R} \rightarrow \partial_{e} N$ (resp. $\gamma: \mathbb{R}_{+} \rightarrow \partial_{e} N$ ) is in tight position with respect to $m$ if $\gamma$ does not contain $m$-waves and the image under $\gamma$ of every unbounded interval intersects $m$.

Lemma 1. If $\gamma: \mathbb{R}_{+} \rightarrow \partial_{e} N$ is a curve which is in tight position with respect to a meridian $m$, then every lift of $\gamma$ to $S^{\prime}$ has a well-defined endpoint in the limit set $\Lambda_{\rho_{0}}$ of the action of $\rho_{0}\left(\pi_{1}(N)\right)$ on $\hat{\mathbb{C}}$.

Proof. Let $\gamma^{\prime}: \mathbb{R}_{+} \rightarrow S^{\prime}$ be a lift of $\gamma$ to $S^{\prime}$. There is a sequence $\left(m_{i}\left(\gamma^{\prime}\right)\right)$ of lifts of $m$ which are intersected by $\gamma^{\prime}$ and indexed by the ordering in $\gamma^{\prime}$. Observe that every lift of $m$ to $S^{\prime}$ separates $S^{\prime}$ and denote by $K_{i}$ the closure in $\hat{\mathbb{C}}$ of the connected component of $\hat{\mathbb{C}}-m_{i}\left(\gamma^{\prime}\right)$ that does not contain $m_{0}\left(\gamma^{\prime}\right)$. Since $\gamma$ is in tight position with respect to $m, K_{i+1}$ is contained in the interior of $K_{i}$ for all $i$ and the diameter of $K_{i}$ tends to zero (compare [Ota88, 1.9, 1.14]).

The following lemma establishes a kind of continuity for the map which associates to lifts of curves $\gamma: \mathbb{R}_{+} \rightarrow \partial_{e} N$ which are in tight position with respect to a common meridian their endpoints in $\Lambda_{\rho_{0}} \subset \hat{\mathbb{C}}$. Notation as in the proof of the last lemma.

Lemma 2. For $j=1, \ldots, \infty$ let $\gamma_{j}: \mathbb{R}_{+} \rightarrow \partial_{e} N$ be curves which are all in tight position with respect to a meridian $m$. Let $m_{0}$ and $\gamma_{j}^{\prime}$ be lifts of $m$ and $\gamma_{j}$ to $S^{\prime}$ with $m_{0}=m_{0}\left(\gamma_{j}^{\prime}\right)$ for all $j=1, \ldots, \infty$. Then the endpoints of $\gamma_{j}^{\prime}$ converge to the endpoint of $\gamma_{\infty}^{\prime}$ if and only if for all $i$ there is $j_{0}$ such that for all $j \geq j_{0}$

$$
m_{i}\left(\gamma_{j}^{\prime}\right)=m_{i}\left(\gamma_{\infty}^{\prime}\right)
$$


So far, we considered general curves on $\partial_{e} N$, we now turn our attention to laminations.

Definition. A lamination is in tight position with respect to a meridian $m$ if every half-leaf is.

Notice that a minimal lamination $\lambda$ is in tight position with respect to a meridian $m$ if and only if some half-leaf in $\lambda$ is since every half-leaf is dense. On the other hand, if a lamination consists of several minimal components each being in tight position with respect to a meridian, it is not clear if there is a common meridian with respect to which all components are in tight position. We prove

Lemma 3. Let $\mu$ be a measured lamination with the property that every component is in tight position with respect to some meridian. Then there is a meridian $m$ intersecting $\mu$ and such that $\mu$ does not contain any $m$-wave.

Proof. Let $\mu_{1}$ be a component of $\mu$ and let $m_{1}$ be a meridian with respect to which $\mu_{1}$ is in tight position. We claim that the set of those homotopy classes of $m_{1}$-waves (rel $m_{1}$ ) that are represented by a subsegment of a leaf of $\mu$ is a finite number $N_{m_{1}}(\mu)$. For this, remark that there are leaves $l_{2}, \ldots, l_{k}$ which are dense in $\mu-\mu_{1}$. This implies that every $m_{1}$-wave in $\mu$ can be represented, up to homotopy $\left(\right.$ rel $\left.m_{1}\right)$, by a segment in one of the leaves $l_{2}, \ldots, l_{k}$. Recall that each of the leaves $l_{2}, \ldots, l_{k}$ is in tight position with respect to some meridian. It follows from lemma 1 that there are, up to homotopy, only finitely many $m_{1}$-waves in $l_{2}, \ldots, l_{k}$. We have proved that $N_{m_{1}}(\mu)<\infty$.

We will inductively reduce this number until we obtain a meridian $m$ with $N_{m}(\mu)=0$. We assume that $N_{m_{1}}(\mu)>0$; hence, there is an $m_{1}$-wave $[a, b]$ contained in one of the leaves $l_{2}, \ldots, l_{k}$ such that $(a, b) \cap m_{1}=\emptyset$. By surgery of $m_{1}$ along $[a, b]$ we obtain meridians $m_{2}, m_{2}^{\prime}$ with the following properties:

(i) $\mu_{1}$ is in tight position with respect to one of the meridians $m_{2}, m_{2}^{\prime}$, say with respect to $m_{2}$, and

(ii) $N_{m_{2}}(\mu) \leq N_{m_{1}}(\mu)-1$.

If $\mu$ contains $m_{2}$-waves, we repeat the process with $m_{2}$. After finitely many steps, we are done.

We finish this section with two results of Otal [Ota88]. In the next subsection, we give complete proofs of more precise statements in the case that $N$ is a handlebody. So, we skip the proofs even though Otal's thèse d'Etat is unfortunately unpublished.

Lemma 4. [Ota88, 1.3] Every minimal component of a lamination $\lambda \in \mathcal{O}$ is in tight position with respect to some meridian.

Definition 1. A leaf $l: \mathbb{R} \rightarrow \partial_{e} N$ of a lamination on $\partial_{e} N$ is called homoclinic if 
there are two sequences $x_{i}, y_{i} \in \mathbb{R}$ and a lift $l^{\prime}$ of $l$ to $S^{\prime}$ such that $\left|x_{i}-y_{i}\right| \rightarrow \infty$ but the distances between $l^{\prime}\left(x_{i}\right)$ and $l^{\prime}\left(y_{i}\right)$ are bounded in $S^{\prime}$.

Otal proved (see the proof of [Ota88, 2.10])

Lemma 5. A lamination $\lambda$ in the Masur-domain is not contained in a lamination with a homoclinic leaf.

\subsection{The handlebody case}

This subsection is devoted to a more detailed analysis of laminations on the boundary of the handlebody. As remarked above, the proofs are inspired by the arguments in [Ota88].

Notice that it is a topological property for a lamination to be an element of $\mathcal{O}$. Denote by $M_{\text {top }}^{\prime}$ the set of those laminations in $\mathcal{P} \mathcal{M L}$ which have same support as laminations in $M^{\prime}$. The main result of this section is the next Proposition which is a slightly stronger version of Lemma 5 in the handlebody case.

Proposition 1. If $N$ is a handlebody and $\mu \subset \partial_{e} N$ is a lamination containing a homoclinic leaf, then every minimal component of $\mu$ is an element of $M_{\text {top }}^{\prime}$.

On the other hand, every Hausdorff-limit of meridians contains a leaf which is homoclinic (see Casson-Long [CL85] or Otal [Ota88]).

Corollary 1. Every minimal component of a Hausdorff-limit of meridians on the boundary of a handlebody is an element of $M_{\text {top }}^{\prime}$.

The proof of Proposition 1 occupies the rest of this section. First, we give three lemmas which help to identify a lamination as an element of $M_{t o p}^{\prime}$.

If $\mu \subset \partial_{e} N$ is a minimal lamination, we denote the smallest compact subsurface of $\partial_{e} N$ containing $\mu$ by $S(\mu)$. It is unique up to isotopy.

Lemma 6. [Ota88, 1.3.2] If $\mu$ is a minimal lamination on the boundary of a handlebody $N$ and $\partial_{e} N-S(\mu)$ is compressible, then $\mu \in M_{\text {top }}^{\prime}$.

Proof. By Dehn's Lemma [Jac80], $\partial_{e} N-S(\mu)$ contains a meridian which bounds a disk in $N$. If the disk is separating, it cuts $N$ into two handlebodies and $\mu$ is contained in one of them, hence there is also a non-separating meridian disjoint from $\mu$. So we assume that $m$ is non-separating. Cut $\partial_{e} N$ along $m$ and join the two resulting boundary components by an embedded arc $\kappa$. The boundary of a regular neighbourhood of $m \cup \kappa$ in $\partial_{e} N$ is a meridian. Since $\kappa$ can be chosen as close to $\mu$ as wanted, we deduce $\mu \in M_{\text {top }}^{\prime}$. 
The proof of Lemma 6 fails to generalize to laminations on the boundary of a general compression body and constitutes the main difference between the handlebody case and the general case in the present context.

By Lemma 4, every component of a measured lamination in $\mathcal{O}$ is in tight position with respect to some meridian. On the other hand we have

Lemma 7. Let $\mu$ be a minimal arational lamination on the boundary of a handlebody. If $\mu$ is not in tight position with respect to any meridian, then $\mu \in M_{\text {top }}^{\prime}$.

Proof. Let $m$ be a meridian and $l$ a leaf of $\mu$. As seen before, if $l$ contains only finitely many homotopy classes $(\mathrm{rel} m$ ) of $m$-waves, then we can find a meridian $m_{0}$ with respect to which $l$ is in tight position. The minimality of $\mu$ then implies that $\mu$ is in tight position with respect to $m_{0}$.

If $l$ contains infinitely many homotopy classes (rel $m$ ) of $m$-waves, it is homoclinic. So, we find sequences $\left(x_{i}\right),\left(y_{i}\right) \subset \mathbb{R}$ such that $l\left(x_{i}\right), l\left(y_{i}\right) \in m$ and the segments $l\left[x_{i}, y_{i}\right]$ are $m$-waves. We may suppose that $l\left(x_{i}\right)$ and $l\left(y_{i}\right)$ converge. Fix a transverse measure on $\mu$. For all $\epsilon>0$ we can find $i, j$ such that the measure of the small subsegments $\left[l\left(x_{i}\right), l\left(x_{j}\right)\right] \subset m$ and $\left[l\left(y_{i}\right), l\left(y_{j}\right)\right] \subset m$ is less than $\epsilon$. The union of these two segments and the $m$-waves $l\left[x_{i}, y_{i}\right]$ and $l\left[x_{j}, y_{j}\right]$ is a compressible curve. By the Loop Theorem [Jac80], we find nearby a meridian $m_{\epsilon}$ with $i\left(\mu, m_{\epsilon}\right)<2 \epsilon$. Taking limits we obtain a lamination $\nu \in M^{\prime}$ with $i(\mu, \nu)=0$ which implies that $\mu$ and $\nu$ have same support as $\mu$ is minimal arational.

We now consider the case that $\mu$ is only minimal. If some component of $\partial S(\mu)$ is a meridian, Lemma 6 shows that $\mu \in M_{t o p}^{\prime}$. If this is not the case, a relative version of the proof of Lemma 7 yields the following Lemma which is essentially a special case of Theorem 1.6 in Otal [Ota88].

Lemma 8. If a minimal lamination $\mu$ on the boundary of a handlebody is not in tight position with respect to any meridian, then $S(\mu)$ is compressible and $\mu \in$ $M_{\text {top }}^{\prime}$

We deduce from Lemma 8 and Lemma 6

Corollary 2. If a minimal component of a lamination $\mu$ on the boundary of a handlebody is not in tight position with respect to any meridian, then every minimal component of $\mu$ is in $M_{\text {top }}^{\prime}$.

Proof of Proposition 1. A similar argument as in the proof of Lemma 7 shows that every $\epsilon$-neighbourhood of a homoclinic leaf $l: \mathbb{R} \rightarrow \partial_{e} N$ in $\mu$ contains a meridian. Lemma 6 implies that every minimal component disjoint from the closure of $l$ is in $M_{\text {top }}^{\prime}$. 
From now on, we assume that $l$ is dense in $\mu$. So $\mu$ has one or two minimal components. If one of them is not in tight position with respect to some meridian, we are done by Corollary 2. Assume that they are in tight position with respect to some meridian. Since a homoclinic leaf is not in tight position with respect to any meridian, the leaf $l$ must be isolated and non-closed. Further, every lift $l^{\prime}$ of $l$ to $S^{\prime}$ has endpoints in the limit set $\Lambda_{\rho_{0}} \subset \hat{\mathbb{C}}$ which coincide since $l$ is homoclinic. If $\mu$ contains two minimal components, then every meridian with respect to which one is in tight position intersects the other. Thus, Lemma 3 provides a meridian $m$ with respect to which every minimal component of $\mu$ is in tight position.

In particular, we find $x_{0}<y_{0} \in \mathbb{R}$ such that the segment $l\left[x_{0}, y_{0}\right]$ is an $m$-wave and such that the half-leaves $\left.l\right|_{\left\{t \leq x_{0}\right\}}$ and $\left.l\right|_{\left\{t \geq y_{0}\right\}}$ are in tight position with respect to $m$. Let $x_{0}>x_{1}>x_{2}>\ldots$ and $y_{0}<y_{1}<y_{2}<\ldots$ be the sequences of all points with $l\left(x_{i}\right) \in l \cap m$ and $l\left(y_{i}\right) \in l \cap m$. We have for all $i<j$ :

(1) The segment $l\left[x_{i}, y_{i}\right]$ is an $m$-wave,

(2) the tangent vectors $\left.\frac{d}{d t} l\right|_{y_{i}}$ and $-\left.\frac{d}{d t} l\right|_{x_{i}}$ point to the same side of $m$, and

(3) the curves $l\left[x_{i}, x_{j}\right]$ and $l\left[y_{i}, y_{j}\right]$ represent the same element in $\pi_{1}(N, D)$ where $D$ is a disk with $\partial D=m$.

We will first treat the case that $\mu$ has only one minimal component $\mu_{0}$. For every train track $\tau$ carrying $\mu=\mu_{0} \cup l$ we will construct a simple closed curve in $M^{\prime}$ carried by $\tau$. As $l$ is not contained in the support of any measured lamination this will prove that $\mu_{0} \in M_{t o p}^{\prime}$.

Suppose first that $d\left(l\left(x_{i}\right), l\left(y_{i}\right)\right) \geq \epsilon>0$ for all $i$. After refining $\tau$, we may assume that every component of $\tau \cap m$ is shorter than $\epsilon$. If two points $a, b$ are contained in the same component of $\tau \cap m$ we denote by $[a, b]$ the subsegment of this component bounded by $a$ and $b$. There are $i<j$ such that one of the following two cases occurs.

Case I:

(1) Each of the pairs $\left\{l\left(x_{i}\right), l\left(x_{j}\right)\right\}$ and $\left\{l\left(y_{i}\right), l\left(y_{j}\right)\right\}$ is contained in a component of $\tau \cap m$,

(2) the tangent vectors $-\left.\frac{d}{d t} l\right|_{x_{i}}$ and $-\left.\frac{d}{d t} l\right|_{x_{j}}$ point to the same side of $m$, and

(3) there is no $k \in\{i+1, \ldots, j-1\}$ such that $l\left(x_{k}\right)$ or $l\left(y_{k}\right)$ belongs to $\left[l\left(x_{i}\right), l\left(x_{j}\right)\right]$.

Case II:

(1) Each of the pairs $\left\{l\left(x_{i}\right), l\left(y_{j}\right)\right\}$ and $\left\{l\left(y_{i}\right), l\left(x_{j}\right)\right\}$ is contained in a component of $\tau \cap m$,

(2) the tangent vectors $-\left.\frac{d}{d t} l\right|_{x_{i}}$ and $\left.\frac{d}{d t} l\right|_{y_{j}}$ point to the same side of $m$, and

(3) there is no $k \in\{i+1, \ldots, j-1\}$ such that $l\left(x_{k}\right)$ or $l\left(y_{k}\right)$ belongs to $\left[l\left(x_{i}\right), l\left(y_{j}\right)\right]$.

Both cases are represented in Figure 1.

If Case I holds, there is no $k$ such that $l\left(x_{k}\right)$ and $l\left(y_{k}\right)$ belong to $\left[l\left(y_{i}\right), l\left(y_{j}\right)\right]$ because $d\left(l\left(x_{k}\right), l\left(y_{k}\right)\right) \geq \epsilon$. Together with the fact that lifts of $l$ to $S^{\prime}$ do not 


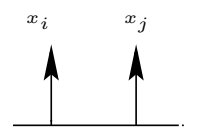

Case I

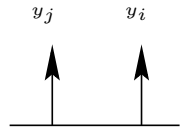

Figure 1

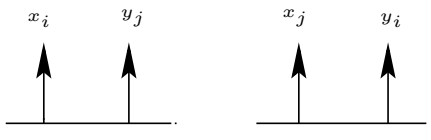

Case II

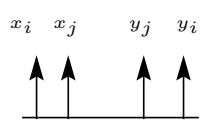

Case I

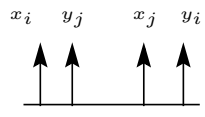

Case II

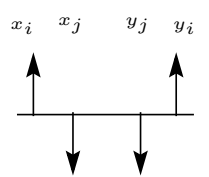

Case III

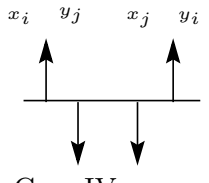

Case IV

Figure 2

intersect and can be compactified to Jordan curves in $\hat{\mathbb{C}}$ this implies that there is no $k \in\{i+1, \ldots, j-1\}$ such that $l\left(x_{k}\right)$ or $l\left(y_{k}\right)$ lies in $\left[l\left(y_{i}\right), l\left(y_{j}\right)\right]$. So, the curves

$$
\gamma_{a}=l\left[x_{i}, x_{j}\right] \cup\left[l\left(x_{i}\right), l\left(x_{j}\right)\right], \quad \eta_{a}=l\left[y_{i}, y_{j}\right] \cup\left[l\left(y_{i}\right), l\left(y_{j}\right)\right]
$$

are homotopic to simple closed geodesics carried by $\tau$. They belong to $M^{\prime}$ since a boundary component of a regular neighbourhood of $\gamma_{a} \cup \eta_{a} \cup l\left[x_{i}, y_{i}\right]$ is a meridian disjoint from $\gamma_{a}$ and $\eta_{a}$.

In Case II, we deduce as in Case I that there is no $k \in\{i+1, \ldots, j-1\}$ such that $l\left(x_{k}\right)$ or $l\left(y_{k}\right)$ belongs to $\left[l\left(y_{i}\right), l\left(x_{j}\right)\right]$. As above this implies that the curve

$$
\gamma_{b}=l\left[x_{i}, x_{j}\right] \cup\left[l\left(x_{j}\right), l\left(y_{i}\right)\right] \cup l\left[y_{i}, y_{j}\right] \cup\left[l\left(y_{j}\right), l\left(x_{i}\right)\right]
$$

is homotopic to a simple closed geodesic carried by $\tau$ and belongs to $M^{\prime}$ because it is disjoint from the boundary component of a neighbourhood of $\gamma_{b} \cup l\left[x_{i}, y_{i}\right]$ which is a meridian.

The arguments are valid for any train track $\tau$. We conclude that the minimal component of $\mu$ is in $M_{\text {top }}^{\prime}$ if $d\left(l\left(x_{i}\right), l\left(y_{i}\right)\right) \geq \epsilon>0$ for all $i$.

Continuing with the assumption that $\mu$ contains only one minimal component $\mu_{0}$, suppose that inf $d\left(l\left(x_{i}\right), l\left(y_{i}\right)\right)=0$. In particular, $l$ contains $m$-waves whose endpoints are close and we cannot directly apply the same arguments as before. Fix $i$ such that $l\left(x_{i}\right), l\left(y_{i}\right)$ are contained in the same component of $\tau \cap m$. If there is no $j>i$ with $l\left(x_{j}\right), l\left(y_{j}\right) \in\left[l\left(x_{i}\right), l\left(y_{i}\right)\right]$ we finish the proof by Lemma 6 as $l\left[x_{i}, y_{i}\right] \cup\left[l\left(x_{i}\right), l\left(y_{i}\right)\right]$ is compressible and disjoint from $\mu_{0}$. Otherwise, let $j>i$ be minimal for the property that $l\left(x_{j}\right)$ and $l\left(y_{j}\right)$ belong to $\left[l\left(x_{i}\right), l\left(y_{i}\right)\right]$. Figure 2 represents the four possible cases.

By the choice of $i, j$ and the fact that lifts of $l$ to $S^{\prime}$ do not intersect and can be compactified to Jordan curves in $\widehat{\mathbb{C}}$, the Cases I and II can be treated as above. The same argument shows in Case III that a boundary component of a regular neighbourhood of

$$
l\left[x_{i}, x_{j}\right] \cup\left[l\left(x_{j}\right), l\left(x_{i}\right)\right] \cup l\left[x_{i}, y_{i}\right] \cup l\left[y_{i}, y_{j}\right] \cup\left[l\left(y_{j}\right), l\left(y_{i}\right)\right]
$$


is homotopic to a simple closed geodesic carried by $\tau$. It is even a meridian since the segments $l\left[x_{i}, x_{j}\right]$ and $l\left[y_{i}, y_{j}\right]$ represent the same element in $\pi_{1}(N, D)$. Similary, in Case IV, the curve $\gamma_{d}$ which is the concatenation of the segments $l\left[x_{i}, x_{j}\right],\left[l\left(x_{j}\right), l\left(y_{i}\right)\right], l\left[y_{i}, x_{i}\right],\left[l\left(x_{i}\right), l\left(y_{j}\right)\right], l\left[y_{j}, y_{i}\right]$ and $l\left[y_{i}, x_{i}\right]$ is homotopic to a meridian carried by $\tau$. As before, we conclude that the minimal component of $\mu$ is in $M_{t o p}^{\prime}$.

The remaining case that $\mu$ has two minimal components can be reduced to the discussion of Case I because there is obviously some $\epsilon>0$ such that $d\left(l\left(x_{i}\right), l\left(y_{j}\right)\right) \geq$ $\epsilon>0$ for all $i, j$. Notice that each of the curves $\gamma_{a}$ and $\eta_{a}$ constructed above approximates one of the minimal components of $\mu$ and is in $M^{\prime}$. This implies that both minimal components of $\mu$ are in $M_{t o p}^{\prime}$.

\section{Morphisms}

This chapter is the central part of the proofs of Theorem 3 and Corollary 3. A large part is inspired by ideas of Skora [Sko96].

$N$ is again a compression body and $\rho_{0}$ is a convex cocompact representation of $\pi_{1}(N)$ which uniformizes $N$.

Definition. Let $T^{\prime}, T$ be $\mathbb{R}$-trees. A morphism from $T^{\prime}$ to $T$ is a map

$$
\Phi: T^{\prime} \longrightarrow T
$$

with the property that every non-degenerate arc $[p, q] \subset T^{\prime}$ contains a nondegenerate subarc $[p, r] \subset[p, q]$ which is mapped isometrically onto $\Phi[p, r] \subset T$.

A morphism is said to fold at a point $p \in T$ and $p$ is a folding point, if there are non-degenerate $\operatorname{arcs}[p, q],\left[p, q^{\prime}\right] \subset T^{\prime},[p, q] \cap\left[p, q^{\prime}\right]=\{p\}$ with $\Phi[p, q]=\Phi\left[p, q^{\prime}\right]$.

\subsection{Morphisms from dual trees}

In section 2, we discussed trees which are dual to measured laminations. If $\mu \in$ $\mathcal{M L}$, the dual tree $T_{\mu}$ can be seen as the leaf space of a measured partial foliation $\mathcal{F}_{\mu}$ enlarging $\mu$ and we denote the projection by $\pi_{\mathcal{F}_{\mu}}: \mathbb{H}^{2} \rightarrow T_{\mu}$. A morphism from a dual tree $T_{\mu}$ to another tree $T$ is said to fold only at complementary regions if the only folding points are projections of complementary regions of $\widetilde{\mathcal{F}}_{\mu} \subset \mathbb{H}^{2}$.

The existence of such morphisms is fundamental in the proof of Skora's Theorem. The following theorem is essentially a special case of the main result in [MO93] (see also [Ota96, chapter 8]).

Theorem (Morgan-Otal). Let $\left(\alpha_{1}, \ldots, \alpha_{3 g-3}\right)$ be a collection of simple closed curves which define a pants decomposition of a closed surface $S$ and let $\pi_{1}(S) \curvearrowright T$ be an action on an $\mathbb{R}$-tree $T$. Then there is a measured lamination $\mu \in \mathcal{M L}$ and 
an equivariant morphism

$$
\Phi: T_{\mu} \longrightarrow T
$$

with $l_{T}\left(\alpha_{i}\right)=l_{T_{\mu}}\left(\alpha_{i}\right)$ for all $i$. Moreover, $\Phi$ folds only at complementary regions.

Remark. Notice that if $l_{T}\left(\alpha_{i}\right)>0$, then $\Phi$ maps the axis of $\alpha_{i}$ in $T_{\mu}$ isometrically to the axis of $\alpha_{i}$ in $T$ [Kap00].

The second step in the proof of Skora's Theorem is to study equivariant morphisms from dual trees $T_{\mu}, \mu \in \mathcal{M L}$, to a tree with a minimal small action of the fundamental group of a closed surface which fold only at complementary regions. Skora proves that such a morphism is an isometry, and in particular $\mu$ is unique. In the present situation, the actions we will consider are not even effective because they factor through the compression homomorphism $\varphi: \pi_{1}\left(\partial_{e} N\right) \rightarrow \pi_{1}(N)$. We prove

Proposition 2. Let $N$ be a compression body with exterior boundary surface $\partial_{e} N$. Let $\pi_{1}(N) \curvearrowright T$ be a minimal small action and let $\mu$ be a measured lamination on $\partial_{e} N$ such that there is an equivariant morphism $\Phi: T_{\mu} \longrightarrow T$ that folds only at complementary regions. Then $\mu$ is not in the Masur domain $\mathcal{O}$.

Moreover, if $N$ is a handlebody, then every minimal component of $\mu$ is an element of $M_{\text {top }}^{\prime}$.

Proof. If some component of $\mu$ is not in tight position with respect to any meridian, then $\mu \notin \mathcal{O}$ by Lemma 4 . Moreover, if $N$ is a handlebody we are done by Corollary 2.

We assume from now on that every component of $\mu$ is in tight position with respect to some meridian. Then by Lemma 3 , there is a meridian $m$ intersecting $\mu$ such that $\mu$ does not contain any $m$-wave. By definition, every component of $\mu$ intersecting $m$ is in tight position with respect to $m$.

We will show that we can extend $\mu$ to a lamination with a homoclinic leaf. In this case, Lemma 5 shows that $\mu \notin \mathcal{O}$. Moreover, Proposition 1 proves that every component of $\mu$ is in $M_{t o p}^{\prime}$ if $N$ is a handlebody.

Lemma 9. $\Phi$ folds along $m$ : for a lift $\widetilde{m}$ of $m$ to $\mathbb{H}^{2}$ there are segments $\tilde{I}_{1}, \tilde{I}_{2} \subset \widetilde{m}$ intersecting in a single point $\tilde{\star}$ such that $\Phi$ maps $\pi_{\mathcal{F}_{\mu}}\left(\tilde{I}_{1}\right)$ and $\pi_{\mathcal{F}_{\mu}}\left(\tilde{I}_{2}\right)$ isometrically onto a non-degenerate segment $J$ in $T$.

Proof. Let $x$ be a point on a lift $\widetilde{m}$ of $m$ to $\mathbb{H}^{2}$. The geodesic $\widetilde{m}$ is invariant under some $\bar{m} \in \pi_{1}\left(\partial_{e} N\right)$ which is trivial in $\pi_{1}(N)$. By equivariance

$$
\Phi\left(\pi_{\mathcal{F}_{\mu}}(\bar{m} x)\right)=\Phi\left(\pi_{\mathcal{F}_{\mu}}(x)\right)
$$


The morphism $\Phi$ folds the non-degenerate segment $\left[\pi_{\mathcal{F}_{\mu}}(x), \pi_{\mathcal{F}_{\mu}}(\bar{m} x)\right]$ only finitely many times. So we can find small segments $\tilde{I}_{1}, \tilde{I}_{2} \subset(x, \bar{m} x)$ such as in the statement.

Remark. Note that the claim is true for every meridian which intersects $\mu$.

We continue with the proof of Proposition 2 and choose a transverse orientation on $m$. For a point $x \in m \cap \mu$ we use the symbol $\mu_{x}^{+}$for the half-leaf of $\mu$ starting at $x$ with positive direction. By definition, $\mu_{x}^{+}$is in tight position with respect to $m$. The following Proposition will be proved at the end of this section.

Proposition 3. For every pair of intervals $\left(I_{1}, I_{2}\right)$ as in Lemma 9, there are $z_{i} \in$ $I_{i} \cap \mu(i=1,2)$ such that the lifts to $S^{\prime}$ of $\mu_{z_{1}}^{+}$and $\mu_{z_{2}}^{+}$have the same endpoints in the limit set $\Lambda_{\rho_{0}}$ of $\rho_{0}\left(\pi_{1}(N)\right)$ in $\hat{\mathbb{C}}$.

We fix a pair of intervals $\left(\tilde{I}_{1}^{0}, \tilde{I}_{2}^{0}\right)$ as in Lemma 9 . As $\Phi$ folds only at complementary regions, the point $\tilde{\star}$ lies in a complementary region $C_{\star}$ of $\widetilde{\mathcal{F}}_{\mu}$. After the collapse of $\mathcal{F}_{\mu}$ to $\mu, C_{\star}$ can be seen as a complementary region of $\tilde{\mu}$, too. There are two well determined boundary leaves $\tilde{\mu}_{1}, \tilde{\mu}_{2}$ of $C_{\star}$ with $\tilde{\mu}_{i} \cap \tilde{I}_{i}^{0} \neq \emptyset$. They are different because separated by the complementary region $C_{\star}$. Up to reversing the orientation, we may assume that $\tilde{\mu}_{1}^{+}$and $\tilde{\mu}_{2}^{+}$are not asymptotic. Next we show that the endpoints in $\Lambda_{\rho_{0}}$ of their projections to $S^{\prime}$ are equal.

We choose a sequence of nested intervals $\left(\tilde{I}_{1}^{k}, \tilde{I}_{2}^{k}\right)_{k \in \mathbb{N}}$ as in Lemma 9 such that $i\left(\tilde{I}_{1}^{k}, \widetilde{\mathcal{F}}_{\mu}\right)=i\left(\tilde{I}_{2}^{k}, \widetilde{\mathcal{F}}_{\mu}\right)>0$ tends to zero. By Proposition 3 , for every $k \in \mathbb{N}$ and $i=1,2$ we get $z_{i}^{k} \in I_{i}^{k} \cap \mu$ such that the lifts to $S^{\prime}$ of $\mu_{z_{1}^{k}}^{+}$and $\mu_{z_{2}^{k}}^{+}$have the same endpoint in the limit set $\Lambda_{\rho_{0}}$.

Since for $i=1,2$ and $k \longrightarrow \infty$ the sequence $i\left(\tilde{I}_{i}^{k}, \widetilde{\mathcal{F}}_{\mu}\right)$ tends to zero, the sequence $\left(\mu_{z_{i}^{k}}^{+}\right)_{k \in \mathbb{N}}$ tends to $\mu_{i}^{+}$, the projection to $\partial_{e} N$ of the boundary half-leaf $\tilde{\mu}_{i}^{+}$. By Lemma 2, the lifts of $\mu_{1}^{+}$and $\mu_{2}^{+}$to $S^{\prime}$ have the same endpoint in $\Lambda_{\rho_{0}}$.

Now let $l$ be the geodesic in $\mathbb{H}^{2}$ joining the endpoints in $\partial \mathbb{H}^{2}$ of $\tilde{\mu}_{1}^{+}$and $\tilde{\mu}_{2}^{+}$. The projection to $\partial_{e} N$ of $\tilde{\mu} \cup l$ is a geodesic lamination $\mu_{l}$ and $l$ projects to a homoclinic leaf, since the endpoints of the projection to $S^{\prime}$ coincide. So, $\mu$ is contained in a lamination with a homoclinic leaf. This finishes the proof of Proposition 2.

The rest of this section is devoted to the proof of Proposition 3.

\subsection{Skora's argument}

We use the same notation as above, $\Phi: T_{\mu} \rightarrow T$ is the morphism of Proposition 2, $\mathcal{F}_{\mu}$ is an enlargement of $\mu, m$ is a meridian with $i(m, \mu)>0$ and such that every 
component of $\mu$ intersected by $m$ is in tight position with respect to it and $\tilde{I}_{1}, \tilde{I}_{2}, \tilde{\star}$ are as in Lemma 9. Denote the projections to the surface $\partial_{e} N$ by $I_{1}, I_{2}, \star$. Recall that $\tilde{\star}$ is in a complementary region $C_{\star}$ of $\widetilde{\mathcal{F}}_{\mu} \subset \mathbb{H}^{2}$.

Fix a transverse orientation on $m$. Take the oriented first return maps of $\mathcal{F}_{\mu}$ [Ota96, Sko96]

$$
A_{i}: I_{i} \cap \mathcal{F}_{\mu} \rightarrow I_{i} \cap \mathcal{F}_{\mu} \text { for } i=1,2 .
$$

$A_{i}$ induces an interval exchange transformation on $\pi_{\mathcal{F}_{\mu}}\left(\tilde{I}_{i}\right) \subset T_{\mu}$. Conjugating $A_{1}, A_{2}$ by $\Phi$ one obtains two interval exchange transformations $B_{1}, B_{2}$ on $J=$ $\left(\Phi \circ \pi_{\mathcal{F}_{\mu}}\right)\left(\tilde{I}_{1}\right)=\left(\Phi \circ \pi_{\mathcal{F}_{\mu}}\right)\left(\tilde{I}_{2}\right) \subset T[$ Sko96].

Let $\mathcal{C}$ denote the free semi-group generated by two letters $c_{1}, c_{2}$ and $\mathcal{B}$ the semi-group generated by the maps $B_{1}, B_{2}$. There is an obvious homomorphism

$$
\theta: \mathcal{C} \longrightarrow \mathcal{B}, \quad c=c_{\epsilon_{1}} \cdot \ldots \cdot c_{\epsilon_{n}} \longmapsto \theta(c)=B_{\epsilon_{n}} \circ \ldots \circ B_{\epsilon_{1}} .
$$

The set of boundary leaves of $\widetilde{\mathcal{F}}_{\mu}$ is countable [Ota96]. A point $z \in T$ such that none of its preimages under $\Phi$ is represented by a boundary leaf of $\widetilde{\mathcal{F}}_{\mu}$ is called regular. If $z \in J$ is a regular point, we denote by $\tilde{z}_{i}$ for $i=1,2$ the point $\tilde{I}_{i} \cap\left(\Phi \circ \pi_{\mathcal{F}_{\mu}}\right)^{-1}(z)$. The projection of $\tilde{z}_{i}$ to $\partial_{e} N$ is denoted $z_{i}$.

A regular point $z \in J$ and a letter $c_{\epsilon} \in\left\{c_{1}, c_{2}\right\}$ determine a closed curve $\gamma_{z, \epsilon} \subset \partial_{e} N$ which is the concatenation of

(1) the subsegment of $I_{\epsilon}$ from $\star$ to $z_{\epsilon}$,

(2) the subsegment of the half-leaf of $\mathcal{F}_{\mu}$ from $z_{\epsilon}$ to $A_{\epsilon} z_{\epsilon}$ in positive direction, and

(3) the subsegment of $I_{\epsilon}$ from $A_{\epsilon} z_{\epsilon}$ to $\star$.

Let $z \in J$ be regular such that for all $B \in \mathcal{B}, B z$ is regular, too. For $c=$ $c_{\epsilon_{1}} \cdot \ldots \cdot c_{\epsilon_{n}} \in \mathcal{C}$ we define the closed curve $\omega_{z}(c)$ in $\partial_{e} N$ to be the concatenation of the curves $\gamma_{\epsilon_{1}, z}, \gamma_{\epsilon_{2}, B_{\epsilon_{1}} z}, \ldots, \gamma_{\epsilon_{n}, B_{\epsilon_{n-1}} \circ \ldots \circ B_{\epsilon_{1}}(z)}$, that is

$$
\omega_{z}(c)=\gamma_{\epsilon_{1}, z} * \gamma_{\epsilon_{2}, B_{\epsilon_{1}}} * \ldots * \gamma_{\epsilon_{n}, B_{\epsilon_{n-1}} \circ \ldots \circ B_{\epsilon_{1}}(z)} .
$$

Recall that $\mathcal{F}_{\mu}$ is obtained from $\mu$ by blowing up closed leaves of $\mu$ to fibered collars. After collapsing the collars back to closed leaves of $\mu$, we denote the image of the curve $\omega_{z}(c)$ by $\omega_{z}(c)$ as well. After a small homotopy near $m$ this curve and $m$ can be made transverse. Moreover, it is in tight position with respect to $m$.

The element in $\pi_{1}\left(\partial_{e} N, \star\right)$ represented by $\omega_{z}(c)$ will be denoted $\left[\omega_{z}(c)\right]$. The map $\left[\omega_{z}(\cdot)\right]: \mathcal{C} \rightarrow \pi_{1}\left(\partial_{e} N, \star\right)$ is injective because $\tilde{\star}$ is in a complementary region [Sko96]. It is not a homomorphism but we have the following equation, which follows from the definition:

$$
\left[\omega_{z}(a \cdot b)\right]=\left[\omega_{z}(a)\right] *\left[\omega_{\theta(a) z}(b)\right] \text { for all } a, b \in \mathcal{C} .
$$

The action of $\varphi\left(\left[\omega_{z}(c)\right]\right) \in \pi_{1}(N)$ on the tree $T$ is related to the interval exchange map $\theta(c)$ by (see [Sko96])

$$
\theta(c)=\varphi\left(\left[\omega_{z}(c)\right]\right) \text { on a small neighbourhood of } z \text { in } J .
$$


Lemma 10. For every pair of intervals $\left(I_{1}, I_{2}\right)$ as in Lemma 9 and every $k$ there is a regular point $z^{k} \in J$ and $a_{1}^{k}=c_{1}^{k} \cdot \ldots, a_{2}^{k}=c_{2}^{k} \cdot \ldots \in \mathcal{C}$ such that the curves $\omega_{z^{k}}\left(a_{1}^{k}\right), \omega_{z^{k}}\left(a_{2}^{k}\right)$ represent the same element in $\pi_{1}(N, \star)$.

The proof of this Lemma is essentially the proof of Proposition 3.1 in [Sko96]. We refer to it for a more detailed exposition.

Proof. Let $z \in J$ be regular such that for all $B \in \mathcal{B}, B z$ is regular, too. We fix $k \in \mathbb{N}$. Let $\mathcal{C}_{n}^{k} \subset \mathcal{C}$ denote the words of length $n$ in the letters $c_{1}^{k}=c_{1} \cdot \ldots \cdot c_{1}$ and $c_{2}^{k}=c_{2} \cdot \ldots \cdot c_{2}$. Cardinality of $\mathcal{C}_{n}^{k}$ is $2^{n}$. As in [Sko96], the cardinality of the sets $\theta\left(\mathcal{C}_{n}^{k}\right)(z)$ only grows polynomially in $n$. So, for fixed $k$ there is a sequence of sets $\left(\mathcal{D}_{n}^{k}\right)_{n}$, with $\mathcal{D}_{n}^{k} \subset \mathcal{C}_{n}^{k}$ such that:

(1) for fixed $n$ any two elements of $\theta\left(\mathcal{D}_{n}^{k}\right)$ map $z$ to the same point in $J$ and

(2) the cardinality of $\mathcal{D}_{n}^{k}$ has exponential growth in $n$.

We fix $d_{n}^{k} \in \mathcal{D}_{n}^{k}$. For fixed $n, k$ the elements of the set $\left(\theta\left(d_{n}^{k}\right)\right)^{-1} \circ \theta\left(\mathcal{D}_{n}^{k}\right)$ are interval exchange maps on $J$ which fix a common segment around $z$.

By smallness of the action $\pi_{1}(N) \curvearrowright T$ and equation (5), the set $\varphi\left(\left[\omega_{z}\left(\mathcal{D}_{n}^{k}\right)\right] *\right.$ $\left.\left[\omega_{z}\left(d_{n}^{k}\right)\right]^{-1}\right)$ is contained in a cyclic subgroup $Z_{n}^{k} \subset \pi_{1}(N)$ [Sko96]. The subgroups can be chosen to satisfy

$$
Z_{n}^{k} \subset Z_{n+1}^{k} \subset \ldots
$$

but such a sequence stabilizes and we find a cyclic subgroup $Z^{k} \subset \pi_{1}(N)$ such that for all $n$

$$
\varphi\left(\left[\omega_{z}\left(\mathcal{D}_{n}^{k}\right)\right] *\left[\omega_{z}\left(d_{n}^{k}\right)\right]^{-1}\right) \subset Z^{k}
$$

As in [Sko96], the cardinality of $\varphi\left(\left[\omega_{z}\left(\mathcal{D}_{n}^{k}\right)\right] *\left[\omega_{z}\left(d_{n}^{k}\right)\right]^{-1}\right)$ has at most linear growth in $n$. So there is $n$ depending on $k$ and there are different words $b_{1}^{k}, b_{2}^{k} \in \mathcal{D}_{n}^{k} \subset \mathcal{C}_{n}^{k}$ with

$$
\varphi\left(\left[\omega_{z}\left(b_{1}^{k}\right)\right]\right)=\varphi\left(\left[\omega_{z}\left(b_{2}^{k}\right)\right]\right)
$$

The words $b_{1}^{k}, b_{2}^{k} \in \mathcal{C}_{n}^{k}$ are different but may coincide at the beginning. Without loss of generality we can assume that

$$
\begin{aligned}
& b_{1}^{k}=\alpha^{k} \cdot c_{1}^{k} \cdot \ldots=\alpha^{k} \cdot a_{1}^{k} \\
& b_{2}^{k}=\alpha^{k} \cdot c_{2}^{k} \cdot \ldots=\alpha^{k} \cdot a_{2}^{k}
\end{aligned}
$$

with $\alpha^{k}$ being a word in $c_{1}^{k}, c_{2}^{k}$ of length less than $n$. By equation (4)

$$
\begin{aligned}
& {\left[\omega_{z}\left(b_{1}^{k}\right)\right]=\left[\omega_{z}\left(\alpha^{k}\right)\right] *\left[\omega_{\theta\left(\alpha^{k}\right) z}\left(a_{1}^{k}\right)\right]} \\
& {\left[\omega_{z}\left(b_{2}^{k}\right)\right]=\left[\omega_{z}\left(\alpha^{k}\right)\right] *\left[\omega_{\theta\left(\alpha^{k}\right) z}\left(a_{2}^{k}\right)\right]}
\end{aligned}
$$

We set $z^{k}=\theta\left(\alpha^{k}\right) z$ and we are done. 
Recall that we have fixed a transverse orientation on $m$ and that for a point $x \in m \cap \mu$ the half-leaf $\mu_{x}^{+}$of $\mu$ starting at $x$ with positive direction is in tight position with respect to $m$.

Proof of Proposition 3. Notation as in Lemma 10. For $i=1,2$ let $\tilde{z}_{i}^{k} \in \tilde{I}_{i} \cap \widetilde{\mathcal{F}}_{\mu}$ be the unique point which is mapped to $z^{k} \in J$ by $\Phi \circ \pi_{\mathcal{F}_{\mu}}$ and $z_{i}^{k}$ the projection to $\partial_{e} N$. After collapsing the collars of $\mathcal{F}_{\mu}$ back to closed leaves of $\mu$ we denote the images of $z_{i}^{k}$ by $z_{i}^{k}$ again (compare above).

In the proof of Lemma 1 , we defined the sequence $\left(m_{j}\right)$ of lifts of $m$ to $S^{\prime}$ for curves which are in tight position with respect to a common meridian. The curves $\omega_{z^{k}}\left(a_{1}^{k}\right)$ and $\omega_{z^{k}}\left(a_{2}^{k}\right)$ provided by Lemma 10 are in tight position with respect to the meridian $m$. Since they represent the same element in $\pi_{1}(N, \star)$

$$
m_{j}\left(\omega_{z^{k}}\left(a_{1}^{k}\right)\right)=m_{j}\left(\omega_{z^{k}}\left(a_{2}^{k}\right)\right) \text { for all } j, k
$$

By construction of the curves $\omega_{z^{k}}\left(a_{i}^{k}\right)$, we have $(i=1,2)$

$$
m_{j}\left(\omega_{z^{k}}\left(a_{i}^{k}\right)\right)=m_{j}\left(\mu_{z_{i}^{k}}^{+}\right) \text {for all } k \text { and } j=1, \ldots, k
$$

Equations (6) and (7) imply

$$
m_{j}\left(\mu_{z_{1}^{k}}^{+}\right)=m_{j}\left(\mu_{z_{2}^{k}}^{+}\right) \text {for all } k \text { and } j=1, \ldots, k
$$

By compactness of $I_{i} \cap \mu$, we can suppose that the sequence $\left(z_{i}^{k}\right)_{k}$ converges to some $z_{i} \in I_{i} \cap \mu$. By Lemma 2, the lifts of $\mu_{z_{1}}^{+}$and $\mu_{z_{2}}^{+}$to $S^{\prime}$ have the same endpoint in the limit set $\Lambda_{\rho_{0}}$.

\section{Realizations}

Let $N$ be a compression body with a convex cocompact hyperbolic structure. Recall that every lamination $\lambda$ in $\mathcal{O}$ is realized by a pleated surface with respect to $\rho_{0}$ and that the induced map from $\lambda$ to the projectivized tangent bundle of $N$ is a homeomorphism onto its image $\mathcal{P}_{\lambda}$.

By definition, the lamination $\lambda \in \mathcal{O}$ is realized in a tree $T$ if there is a continuous and equivariant map from $\widetilde{\mathcal{P}}_{\lambda}$, the lift of $\mathcal{P}_{\lambda}$ to the projectivized tangent bundle of $\mathbb{H}^{3}$, to the tree $T$ which is injective when restricted to any leaf.

Since $\lambda$ is mapped homeomorphically onto $\mathcal{P}_{\lambda}$ it suffices to find a continuous and $\pi_{1}\left(\partial_{e} N\right)$-equivariant map from $\tilde{\lambda}$, the lift of $\lambda$ to $\mathbb{H}^{2}$, to the tree $T$ which is injective when restricted to any leaf. By abuse, if such a map exists we will also say that $\lambda$ is realized in $T$.

Theorem 3. Let $\pi_{1}(N) \curvearrowright T$ be a non-trivial minimal small action on an $\mathbb{R}$-tree $T$ and $\lambda$ a minimal arational measured lamination in the Masur domain, then $\lambda$ is realized in $T$. 
The idea of the proof is to show that there is a measured lamination $\mu$ on the exterior boundary $\partial_{e} N$ and a morphism $\Phi_{\mu}: T_{\mu} \rightarrow T$ such that the composition of the projection $\mathbb{H}^{2} \rightarrow T_{\mu}$ with $\Phi_{\mu}$ can be deformed to a realization of $\lambda$ in $T$.

Proof. We choose a sequence of simple closed curves $\left(\gamma_{i}\right)_{i}$ converging to $\lambda$ with respect to the Hausdorff topology. By Morgan's and Otal's Theorem, for all $i$, there is a measured lamination $\mu_{i}$ with $i\left(\mu_{i}, \gamma_{i}\right)=l_{T_{\mu_{i}}}\left(\gamma_{i}\right)=l_{T}\left(\gamma_{i}\right)$ and a morphism

$$
\Phi_{\mu_{i}}: T_{\mu_{i}} \rightarrow T \text {. }
$$

Now let us show that $l_{T}\left(\gamma_{i}\right)>0$ for large $i$. Up to choice of a subsequence, we may assume that the supports of the laminations $\mu_{i}$ converge in the Hausdorff topology to a lamination $\mu_{\mathcal{H}}$. By Proposition 2, the measured laminations $\mu_{i}$ are not in the Masur domain $\mathcal{O}$. This implies that the Hausdorff limit $\mu_{\mathcal{H}}$ cannot contain a lamination in $\mathcal{O}$. In particular, the minimal arational lamination $\lambda$ intersects $\mu_{\mathcal{H}}$. So, for all but finitely many $i$, say for all, one has

$$
l_{T}\left(\gamma_{i}\right)=i\left(\mu_{i}, \gamma_{i}\right)>0
$$

Remark that the morphism $\Phi_{\mu_{i}}$ maps isometrically the axis of every element in the conjugacy class represented by $\gamma_{i}$.

For all $i$, we choose an enlargement $\mathcal{F}_{\mu_{i}}$ of $\mu_{i}$ in order to obtain a continuous and equivariant projection $\pi_{\mathcal{F}_{\mu_{i}}}: \mathbb{H}^{2} \rightarrow T_{\mu_{i}}$. We may assume that the enlargements $\mathcal{F}_{\mu_{i}}$ converge in the Hausdorff topology to the lamination $\mu_{\mathcal{H}}$.

The map $\Phi_{\mu_{i}} \circ \pi_{\mathcal{F}_{\mu_{i}}}$ is continuous, equivariant and, by equation (8), it is monotone on every lift of $\gamma_{i}$ to $\mathbb{H}^{2}$. Here, a map from an interval to a tree is monotone if the preimage of every point is at most a bounded interval [Ota96].

Lemma 11. There is a train track $\tau$ which carries $\lambda$ such that, for large $i$, the $\operatorname{map} \Phi_{\mu_{i}} \circ \pi_{\mathcal{F}_{\mu_{i}}}$ satisfies:

(i) It is constant on ties, and monotone and non-constant on the rails of every lift $\widetilde{R}_{j} \subset \mathbb{H}^{2}$ of every rectangle $R_{j} \subset \tau$.

(ii) The images of any two rectangles $\widetilde{R}_{j}, \widetilde{R}_{k} \subset \mathbb{H}^{2}$ which meet in vertical sides intersect in exactly one point.

Proof. Since $\lambda$ intersects $\mu_{\mathcal{H}}$, the construction in [Ota96, chapter 3] yields a train track $\tau$ carrying $\lambda$ such that, for large $i$, the projection $\pi_{\mathcal{F}_{\mu_{i}}}: \mathbb{H}^{2} \rightarrow T_{\mu_{i}}$ is constant on ties, and monotone and non-constant on the rails of every lift $\widetilde{R}_{j} \subset \mathbb{H}^{2}$ of every rectangle $R_{j} \subset \tau$.

We may assume that $\tau$ carries $\lambda$ minimally, i.e. for any two rectangles $\widetilde{R}_{j}, \widetilde{R}_{k} \subset$ $\mathbb{H}^{2}$ which meet in vertical sides one has $\widetilde{R}_{j} \cap \widetilde{R}_{k} \cap \tilde{\lambda} \neq \emptyset$. For large $i$, the train track $\tau$ carries the curve $\gamma_{i}$ minimally, too. We are going to show that for all such $i$, the map $\Phi_{\mu_{i}} \circ \pi_{\mathcal{F}_{\mu_{i}}}$ satisfies (i) and (ii).

Given a rectangle $\widetilde{R}_{j}$, there is a lift $\tilde{\gamma}_{i} \subset \mathbb{H}^{2}$ of $\gamma_{i}$ crossing $\widetilde{R}_{j}$; hence, we can homotope along ties the rails of $\widetilde{R}_{j}$ into the lift $\tilde{\gamma}_{i}$. As remarked above, the map 
$\Phi_{\mu_{i}} \circ \pi_{\mathcal{F}_{\mu_{i}}}$ is monotone on $\tilde{\gamma}_{i}$, and thus on the rails of $\widetilde{R}_{j}$. It is not constant because $\pi_{\mathcal{F}_{\mu_{i}}}\left(\widetilde{R}_{j}\right)$ is a non-degenerate segment and $\Phi_{\mu_{i}}$ is a morphism. Property (i) follows.

Given two rectangles $\widetilde{R}_{j}, \widetilde{R}_{k} \subset \mathbb{H}^{2}$ which meet in vertical sides there is a lift $\tilde{\gamma}_{i} \subset \mathbb{H}^{2}$ of $\gamma_{i}$ with $\tilde{\gamma}_{i} \cap \widetilde{R}_{j} \cap \widetilde{R}_{k} \neq \emptyset$. Again, since $\Phi_{\mu_{i}} \circ \pi_{\mathcal{F}_{\mu_{i}}}$ is monotone on $\tilde{\gamma}_{i}$, property (ii) follows.

For $\tau$ and $i$ as in lemma 11, it follows from [Ota96, 3.1.5, 3.1.6] that the map $\Phi_{\mu_{i}} \circ \pi_{\mathcal{F}_{\mu_{i}}}$ can be homotoped to a realization in $T$ of every lamination carried by $\tau$, in particular of $\lambda$. This concludes the proof of Theorem 3 .

In the particular case that $N$ is a handlebody we obtain

Theorem 4. Let $N$ be a handlebody and $\pi_{1}(N) \curvearrowright T$ a non-trivial minimal small action on an $\mathbb{R}$-tree $T$. Further let $\lambda_{0}$ be a minimal component of a lamination $\lambda$ in the Masur domain.

Either $\lambda_{0}$ is realized in $T$ or there is a train track $\tau_{0}$ carrying $\lambda_{0}$ and a continuous and equivariant map $\Phi_{0}: \tilde{\tau}_{0} \rightarrow T$ that maps every connected component of $\tilde{\tau}_{0}$ to a point.

Proof. If $\lambda_{0}$ is a simple closed geodesic, then there is nothing to prove. So suppose it is not.

As above choose a sequence of simple closed curves $\left(\gamma_{i}\right)_{i}$ converging to $\lambda_{0}$ with respect to the Hausdorff topology. Again, for all $i$, there is a measured lamination $\mu_{i}$ with $i\left(\mu_{i}, \gamma_{i}\right)=l_{T_{\mu_{i}}}\left(\gamma_{i}\right)=l_{T}\left(\gamma_{i}\right)$ and a morphism

$$
\Phi_{\mu_{i}}: T_{\mu_{i}} \rightarrow T
$$

Suppose that the laminations $\mu_{i}$ converge in the Hausdorff topology to a lamination $\mu_{\mathcal{H}}$. Every minimal component of $\mu_{\mathcal{H}}$ is contained in the Hausdorff limit of components of $\mu_{i}$, thus by Proposition 2 in a Hausdorff limit of laminations in $M_{t o p}^{\prime}$ and so in a Hausdorff limit of meridians. By Corollary 1, every minimal component of $\mu_{\mathcal{H}}$ is an element of $M_{t o p}^{\prime}$.

So either $\lambda_{0}$ is transverse to $\mu_{\mathcal{H}}$ or disjoint from $\mu_{\mathcal{H}}$. In the first case we conclude as in the proof of Theorem 3. If $\lambda_{0} \cap \mu_{\mathcal{H}}=\emptyset$, choose a train track $\tau_{0}$ carrying $\lambda_{0}$ and disjoint from $\mu_{\mathcal{H}}$. For $i$ large enough, there is a partial foliation $\mathcal{F}_{\mu_{i}}$ enlarging the measured lamination $\mu_{i}$ such that $\tau_{0}$ is also disjoint from $\mathcal{F}_{\mu_{i}}$. This implies that the image of every connected component of $\tilde{\tau}_{0}$ is mapped by $\pi_{\mathcal{F}_{\mu_{i}}}$ to a point in $T_{\mu_{i}}$. Define $\Phi_{0}$ to be $\Phi_{\mu_{i}} \circ \pi_{\mathcal{F}_{\mu_{i}}}$. 
Corollary 3. Let $N$ be a handlebody and $\pi_{1}(N) \curvearrowright T$ be a non-trivial minimal small action on an $\mathbb{R}$-tree $T$. At least one minimal component of every measured lamination in the Masur domain is realized in $T$.

Proof. Suppose the claim is false. By Theorem 4, every minimal component $\lambda_{j}$ $(j=1, \ldots, k)$ of $\lambda$ is carried by a train track $\tau_{j}$ and there is a continuous and equivariant map $\Phi_{j}: \tilde{\tau}_{j} \rightarrow T$ that maps every connected component of $\tilde{\tau}_{j}$ to a single point. After refining the train tracks, we may assume that they are pairwise disjoint.

Since $\mathcal{O}$ is open there are simple closed curves $\eta_{j}$ carried by $\tau_{j}$ such that the multicurve $\eta=\eta_{1} \cup \cdots \cup \eta_{k}$ is in the Masur domain. By Morgan's and Otal's Theorem, we find a measured lamination $\mu$ and a morphism $\Phi_{\mu}: T_{\mu} \rightarrow T$ such that $0=l_{T}\left(\eta_{j}\right)=l_{T_{\mu}}\left(\eta_{j}\right)=i\left(\mu, \eta_{j}\right)$ for all $j=1, \ldots, k$. This contradicts the fact that by Proposition 2 every minimal component of $\mu$ is an element of $M_{\text {top }}^{\prime}$ whence $i(\mu, \eta)>0$.

\section{References}

[And98] J. W. Anderson, A brief survey of the deformation theory of Kleinian groups, in: The Epstein Birthday Schrift, Geom. Topol. Monogr. 1 (1998), 23-49.

[Bes88] M. Bestvina, Degenerations of the hyperbolic space, Duke Math. J. 56 (1988), 143161.

[Bon83] F. Bonahon, Cobordism of automorphisms of surfaces, Ann. Sci. Ec. Norm. Super., IV. Ser. 16 (2) (1983), 237-270.

[Can91] R. D. Canary, The Poincaré metric and a conformal version of a theorem of Thurston, Duke Math. J. 64 (2) (1991), 349-359.

[Can93] R. D. Canary, Algebraic convergence of Schottky groups, Trans. Am. Math. Soc., 337 (1993), 235-258.

[CB88] A. J. Casson and S. A. Bleiler, Automorphisms of surfaces after Nielsen and Thurston, Number 9 in LMS Student Texts, Cambridge University Press, 1988.

[CL85] A. J. Casson and D. D. Long, Algorithmic compression of surface automorphisms, Invent. math. 81 (2) (1985), 295-303.

[CV91] M. Culler and K. Vogtmann, The boundary of outer space in rank two, in: Arboreal group theory, 189-230, Math. Sci. Res. Inst., 1991.

[FLP79] A. Fathi, F. Laudenbach and V. Poénaru, Travaux de Thurston sur les surfaces, Astérisque 66-67 (1979).

[GL95] D. Gaboriau and G. Levitt, The rank of actions on $\mathbb{R}$-trees, Ann. Sci. Ec. Norm. Supér. 28 (5) (1995), 549-570.

[Jac80] W. Jaco, Lectures on three-manifold topology, Number 43 in Conference Board of the Mathematical Sciences, Regional Conference Series in Mathematics, American Mathematical Society, 1980.

[Kap00] M. Kapovich, Hyperbolic manifolds and discrete groups, Progress in Mathematics 183, Birkhäuser, 2000.

[Mas86] H. Masur, Measured foliations and handlebodies, Ergodic Theory Dyn. Syst. 6 (1986), 99-116.

[Mas88] B. Maskit, Kleinian groups, Grundlehren der Mathematischen Wissenschaften 287, Springer-Verlag, 1988. 
[MO93] J. W. Morgan and J.-P. Otal, Relative growth rates of closed geodesics on a surface under varying hyperbolic structures, Comment. Math. Helv. 68 (1993), 171-208.

[MS84] J. W. Morgan and P. B. Shalen, Degenerations of hyperbolic structures I: Valuations, trees and surfaces, Ann. Math., II. Ser. 120 (1984), 401-476.

[MT98] K. Matsuzaki and M. Taniguchi, Hyperbolic Manifolds and Kleinian Groups, Oxford Mathematical Monographs, Oxford Science Publications, 1998.

[Ohs] K. Ohshika, Kleinian groups which are limits of geometrically finite groups, preprint.

[Ohs97] K. Ohshika, A convergence theorem for Kleinian groups which are free products, Math. Ann. 3091 (1997), 53-70.

[Ota88] J.-P. Otal, Courants géodésiques et produits libres, Thèse d'Etat, Université ParisSud, Orsay, 1988.

[Ota94] J.-P. Otal, Sur la dégénérescence des groupes de Schottky, Duke Math. J. 74 (1994), 777-792.

[Ota96] J.-P. Otal, Le théorème d'hyperbolisation pour les variétés fibrées de dimension 3 , Astérisque 235 (1996).

[Pau88] F. Paulin, Topologie de Gromov équivariante, structures hyperboliques et arbres réels, Invent. math. 94 (1988), 53-80.

[Sko96] R. K. Skora, Splittings of surfaces, J. Am. Math. Soc. 9 (2) (1996), 605-616.

[Thu86] W. P. Thurston, Hyperbolic Structures on 3-manifolds, I, II, III, preprint, 1986.

Gero Kleineidam

Universität Bonn

Mathematisches Institut

Beringstr. 1

D-53115 Bonn

Germany

e-mail: Gero.Kleineidam@math.uni-bonn.de
Juan Souto

Universität Bonn

Mathematisches Institut

Beringstr. 1

D-53115 Bonn

Germany

e-mail: souto@math.uni-bonn.de

(Received: November 30, 2000)

(17) To access this journal online:

(ID) http://www.birkhauser.ch 